\title{
Stabilizability diagram for turning processes subjected to digital PD control
}

\author{
David Lehotzky • Janos Turi • Tamas Insperger
}

Received: 10 October 2013 / Revised: 19 November 2013 / Accepted: 22 November 2013 / Published online: 10 December 2013

(C) Springer-Verlag Berlin Heidelberg 2013

\begin{abstract}
Stabilizability of the turning process subjected to a digital proportional-derivative controller is analyzed. The governing equation involves a term with continuous-time point delay due to the regenerative effect and terms with piecewise-constant arguments due to the zero-order hold of the digital control. The resulting hybrid system can be represented as a delay-differential equation with time-periodic delay, for which the stability properties are analyzed using the semi-discretization method. The critical depth of cut is determined, which limits the stabilizability of the machining process for a given spindle speed in the sense that machining operation at larger than the critical depth of cut cannot be stabilized by the applied digital controller for a fixed sampling period. The resulted stabilizability diagram shows some similarities to the traditional stability lobe diagram of machining processes.
\end{abstract}

Keywords Machine tool chatter - Regenerative delay · PD controller · Sampling effect · Semi-discretization . Stabilizability

D. Lehotzky $\cdot$ T. Insperger $(\bowtie)$

Department of Applied Mechanics, Budapest University

of Technology and Economics, Budapest 1521, Hungary

e-mail: insperger@mm.bme.hu

D. Lehotzky

e-mail: lehotzky@mm.bme.hu

J. Turi

Programs in Mathematical Sciences, University of Texas at Dallas,

Richardson, TX 75083, USA

e-mail: turi@utdallas.edu

\section{Introduction}

Machine tool chatter is the large amplitude vibration between the tool and the workpiece involving intermittent loss of contact. Chatter is deteriorative to the machining process: it results in poor workpiece surface quality, increases the tool wear and can even damage the tool. Since the pioneering work of Tobias and Tlusty $[1,2]$, the most commonly accepted explanation for chatter is the so-called regenerative effect $[3,4]$ : the cutting edge interferes with its own past oscillation recorded on the wavy surface cut on the workpiece. For turning processes, the tool cuts the surface that was modulated by the tool one turn earlier. For milling processes, the cutting edge cuts the surface that was modulated by the previous cutting edge one tooth-pass period earlier. In the next passage of the cutting edge these vibration marks generate further undulations of the tool-workpiece system. Due to its hereditary nature, this phenomenon can be described by delay-differential equations (DDEs).

In the manufacturing community, the stability properties of machining processes are usually depicted by the socalled stability lobe diagrams (SLDs), which plot the maximum stable depths of cut versus the spindle speed. These diagrams provide a guide to the machinist to select the optimal technological parameters in order to achieve maximum material removal rate without chatter [5-7]. The available chatter-free technological parameters however often do not allow the full utilization of the capacity of the machine center. Machining at high spindle speeds with large depths of cut, which the machine tool structure and the spindle are capable of, are often limited by chatter. In order to operate the machine in these regions, chatter suppression techniques should be applied. These techniques show a big variety including passive, semi-active and active methods. 
One semi-active strategy is to perturb the time delay of the process by continuously modulating the spindle speed [8-11]. The advantage of this method is that the frequency and amplitude of the spindle speed variation can easily be adjusted in computer numerical control (CNC) machines even during the machining process. The disadvantage of the method is that it can be applied only for low spindle speeds, since its application is limited by the actuation power and the inertia of the spindle. Another concept is the application of passive chatter control techniques such as vibration absorbers or passive dampers [12-15]. Advantages of these techniques are that they are cheap, they do not require external energy and they never destabilize a stable system. Drawbacks are that limited amount of damping can be achieved, and it is not necessarily concentrated on the critical modes of the system. They should accurately be tuned to the system and they are not robust against changing machining conditions. Active chatter control techniques aim at reducing chatter vibrations by applying damping to the spindle or the tool in an active way, thus lifting the stability lobe diagrams around a specific spindle speed [16-18]. Several actuators can be applied, such as active magnetic bearings [19,20], piezo-electric [21,22] and electro-hydraulic actuators [23]. These techniques are also called alteration of the spindle dynamics, because the underlying control algorithm can be considered as a kind of artificial dynamics, which is coupled to the original toolworkpiece system. For instance, in an ideal single-degree-offreedom case, a velocity feedback presents a clear damping with the feedback gain representing the artificial damping parameter. Practical realizations of the control system, however, involve implementation imperfections. In case of digital control, the sampling effect attributes an intermittent dynamics to the otherwise continuous-time system. This result in a hybrid system involving terms with point delay and terms with piecewise-constant arguments in the governing equations [24]. Also, the control loop involves feedback delays which may interfere with the regenerative delay of the system. Note that for high-speed machining operations, the tooth passing frequency and the natural frequencies of the spindle are in the region of the sampling frequency of the controller.

It is known that stabilization by digital feedback has limitations. For a simple inverted pendulum controlled by a digital proportional-derivative (PD) controller with a fixed sampling period, there is a critical length for stabilizability. If the pendulum is shorter than this critical length, then it cannot be balanced by the PD controller [25]. The goal of this paper is to determine the limit of stabilizability of machining processes in a similar sense as it is used for the inverted pendulum. We consider a simple two-degree-of-freedom model of a turning process subjected to a digital PD controller. As opposed to the inverted pendulum, where the open loop system is described by an ordinary differential equation (ODE), in case of chatter control, the open loop system is already described by a DDE due to the regenerative effect, which presents an extra difficulty in the stability analysis. The stability of this hybrid system is analyzed using the third-order two-point semi-discretization method according to [24,26]. As a main contribution of this paper, we introduce the stabilizability diagram, which shows the maximum depths of cut versus the spindle speed that can be achieved by a digital PD controller with a fixed sampling period. The outline of the paper is as follows. First the mechanical model is presented in Sect. 2. Then the stabilizability of the hybrid system is investigated using the semi-discretization method in Sect. 3. The stabilizability diagram is presented in Sect. 4. Finally, the results are concluded in Sect. 5.

\section{Mechanical model}

A sketch of the turning process can be seen in Fig. 1. Since the stiffness of the tool in direction $z$ is orders of magnitudes higher than in directions $x$ and $y$, therefore vibrations of the tool in direction $z$ can be neglected and the cutting process can be described with a two-degree-of-freedom model, shown in Fig. 2. The governing equations of the tool are given as

$$
\begin{aligned}
& m \ddot{x}(t)+c_{x} \dot{x}(t)+k_{x} x(t)=F_{x}(t), \\
& m \ddot{y}(t)+c_{y} \dot{y}(t)+k_{y} y(t)=F_{y}(t)-Q(t),
\end{aligned}
$$

where $m$ is the modal mass of the tool, $c_{x}, c_{y}$ and $k_{x}, k_{y}$ are the modal damping and stiffness of the tool in the tangential $(x)$ and normal $(y)$ directions, respectively, $F_{x}(t)$ and $F_{y}(t)$ are the $x$ and $y$ components of the cutting force and $Q(t)$ is the control force. The formula of the cutting force can be given by the empirical power law

$$
\begin{aligned}
& F_{x}(t)=K_{x} w h^{q}(t), \\
& F_{y}(t)=K_{y} w h^{q}(t),
\end{aligned}
$$

where $K_{x}, K_{y}$ are the cutting force coefficients in the tangential and the normal directions, respectively, $w$ denotes the chip width (which is equal to the depth of cut in this

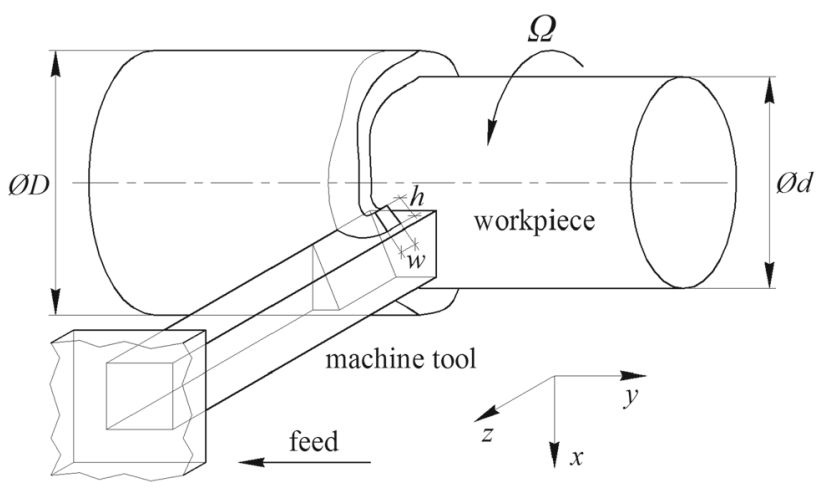

Fig. 1 Chip removal in orthogonal turning 


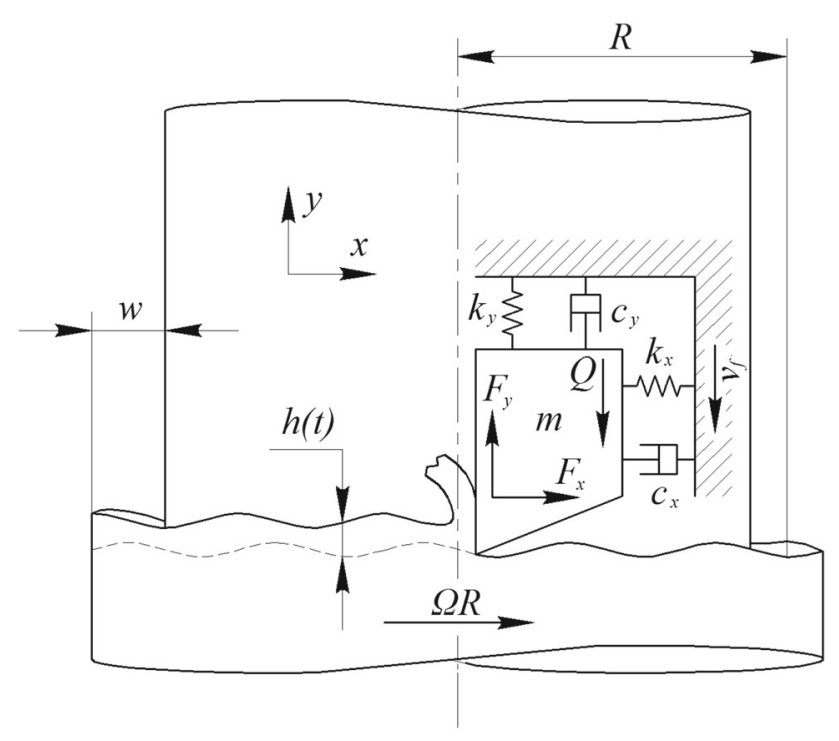

Fig. 2 Two-degree-of-freedom model of the digitally controlled turning process

case), $h(t)$ is the instantaneous chip thickness and $q$ is the cutting force exponent. We assume that the vibration amplitudes in the tangential direction are significantly smaller than the diameter of the workpiece, thus the regenerative delay is considered to be constant [27]. The chip thickness can be given as

$h(t)=v_{f} \tau+y(t-\tau)-y(t)$,

where $v_{f}$ is the feed velocity and $\tau=60 / \Omega$ the regenerative delay (the rotation period of the workpiece) with $\Omega$ being the spindle speed in [rpm]. Note, that if $h<0$, then the contact between the cutting tool and the workpiece ceases, thus the cutting force is zero. This results that the turning process is a non-smooth dynamical system. It is assumed throughout this work, that the tool never leaves the workpiece, that is $h(t) \geq 0$ for all $t$. The governing equations are non-linear DDEs of the form

$$
\begin{aligned}
m \ddot{x}(t)+c_{x} \dot{x}(t)+k_{x} x(t)= & K_{x} w\left(v_{f} \tau+y(t-\tau)-y(t)\right)^{q}, \\
m \ddot{y}(t)+c_{y} \dot{y}(t)+k_{y} y(t)= & K_{y} w\left(v_{f} \tau+y(t-\tau)-y(t)\right)^{q} \\
& -Q(t) .
\end{aligned}
$$

Clearly, the stability of the second equation determines the stability of the system, since the first equation is a simple stable oscillator with a forcing term. Therefore, we analyze only Eq. (7). The desired position of the tool is the trivial equilibrium $y_{\mathrm{e}}=K_{y} w\left(v_{f} \tau\right)^{q} / k_{y}$ of the uncontrolled system. Linearisation about this trivial equilibrium gives

$\ddot{\eta}(t)+2 \zeta \omega_{\mathrm{n}} \dot{\eta}(t)+\left(\omega_{\mathrm{n}}^{2}+H\right) \eta(t)=H \eta(t-\tau)-\frac{1}{m} Q(t)$, where $\omega_{\mathrm{n}}$ and $\zeta$ are the natural angular frequency and relative damping of the tool in direction $y, H=K_{y} w q\left(v_{f} \tau\right)^{q-1} / m$ is the specific cutting force coefficient and $\eta(t)=y(t)-y_{\mathrm{e}}$ is the perturbation about the trivial equilibrium of the tool in direction $y$. Note that in this model, we concentrate only on the regenerative effect, but other phenomena, such as friction or thermal effects, may also contribute to the dynamics of the system [28].

A digital PD controller is assumed, which involves a zero-order hold and a processing delay. Measurement of the position and the velocity is made at each sampling instant $t_{j}=j \Delta t, j \in \mathbb{Z}$. We assume that the processing time, which is the necessary time for the calculation of the control force, is less than the length $\Delta t$ of the sampling period. The control force is calculated using the most recent available measurements and is kept constant over the subsequent sampling period. It is assumed, that the position and the velocity is measured without any noise or error. Thus the control force can be given as

$$
Q(t)=k_{\mathrm{p}}\left(y\left(t_{j-1}\right)-y_{\mathrm{e}}\right)+k_{\mathrm{d}} \dot{y}\left(t_{j-1}\right), \quad t \in\left[t_{j}, t_{j+1}\right),
$$

where $k_{\mathrm{p}}$ and $k_{\mathrm{d}}$ are the proportional and derivative control gains, respectively. Substitution to Eq. (8) gives

$$
\begin{aligned}
\ddot{\eta}(t) & +2 \zeta \omega_{\mathrm{n}} \dot{\eta}(t)+\left(\omega_{\mathrm{n}}^{2}+H\right) \eta(t) \\
= & H \eta(t-\tau)-\frac{1}{m}\left(k_{\mathrm{p}} \eta\left(t_{j-1}\right)+k_{\mathrm{d}} \dot{\eta}\left(t_{j-1}\right)\right), \\
& t \in\left[t_{j}, t_{j+1}\right) .
\end{aligned}
$$

Equation (8) can be further generalized by the introduction of dimensionless time $T=\omega_{\mathrm{n}} t$, resulting

$$
\begin{aligned}
& \eta^{\prime \prime}(T)+2 \zeta \eta^{\prime}(T)+\left(1+H_{\mathrm{d}}\right) \eta(T) \\
& =H_{\mathrm{d}} \eta\left(T-\tau_{\mathrm{d}}\right)-K_{\mathrm{p}} \eta\left(T_{j-1}\right)-K_{\mathrm{d}} \eta^{\prime}\left(T_{j-1}\right), \\
& \quad T \in\left[T_{j}, T_{j+1}\right),
\end{aligned}
$$

where $H_{\mathrm{d}}=H / \omega_{\mathrm{n}}^{2}$ is the dimensionless specific cutting force coefficient, $\tau_{\mathrm{d}}=\omega_{\mathrm{n}} \tau=2 \pi / \Omega_{\mathrm{d}}$ is the dimensionless time delay with $\Omega_{\mathrm{d}}=2 \pi \Omega /\left(60 \omega_{\mathrm{n}}\right)$ denoting the dimensionless spindle speed, $K_{\mathrm{p}}=k_{\mathrm{p}} /\left(m \omega_{\mathrm{n}}^{2}\right), K_{\mathrm{d}}=k_{\mathrm{p}} /\left(m \omega_{\mathrm{n}}\right)$ are the dimensionless proportional and derivative control gains and $T_{j}=j \omega_{\mathrm{n}} \Delta t$ are the dimensionless sampling instants. Since $H_{\mathrm{d}}$ is proportional to the depth of cut, we will refer this parameter as dimensionless depth of cut.

Equation (11) defines a hybrid system in the sense that involves two different types of delays. The term $\eta\left(T-\tau_{\mathrm{d}}\right)$ defines a continuous-time point delay of the state (state delay), while the terms $\eta\left(T_{j-1}\right)$ and $\eta^{\prime}\left(T_{j-1}\right)$ with piecewiseconstant arguments present a discrete-time delay in the feedback (feedback delay). The latter terms can be represented as terms with periodic time delay in the form $\eta(T-\rho(T))$ and $\eta^{\prime}(T-\rho(T))$, where the time delay is a piecewise-linear 


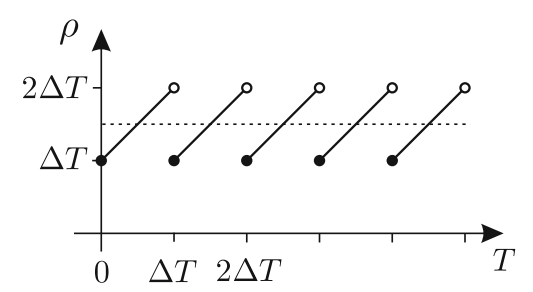

Fig. 3 Representation of the sampling effect as time-varying delay

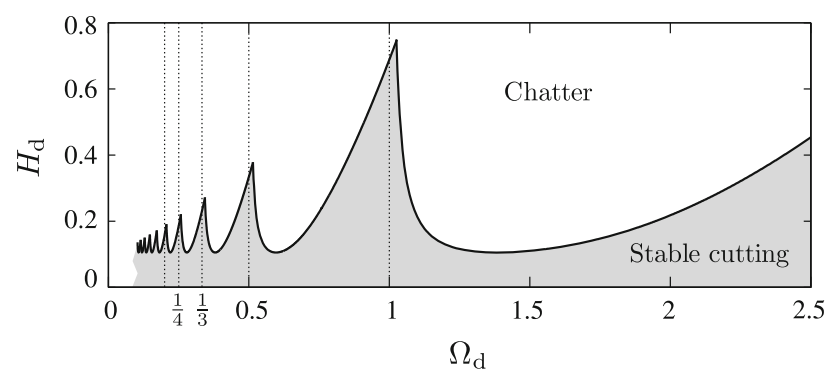

Fig. 4 Dimensionless SLD of the uncontrolled turning process for $\zeta=$ 0.05

function given as $\rho(T)=T+\Delta T-T_{j}, T \in\left[T_{j}, T_{j+1}\right)$ (see Fig. 3). According to this interpretation, sampling in the feedback loop presents a parametric excitation in the time delay and the period of the parametric excitation is equal to the sampling period $\Delta T$. Consequently, the governing equation is a periodic DDE, and the stability analysis can be performed according to the Floquet theory of DDEs. In the recent decades, several numerical and semi-analytical techniques have been developed for the stability analysis of periodic DDEs, such as the semi-discretization method $[29,30]$, the multi-frequency solution [31], the pseudospectral collocation method [32], the continuous time approximation [33,34], the spectral element method [35], the method of characteristic matrices [36], the construction of characteristic function using Fredholm theory [37], the subspace iteration technique or the extended multi-frequency solution [7], to mention just a few. Here, we will use the semi-discretization method with small enough discretization step to capture the interplay of the sampling effect and the regenerative delay.

The stability properties of the uncontrolled case, described by Eq. (11) with $Q(T)=0$, can be determined analytically by the D-subdivision method [38]. The well-known SLD is shown in Fig. 4.

\section{Stabilizability analysis}

Before analyzing the hybrid system given by Eq. (11), the limits of the stabilizability of unstable systems by digital feedback control is demonstrated using an unstable secondorder system without state delay, namely the inverted pendulum.

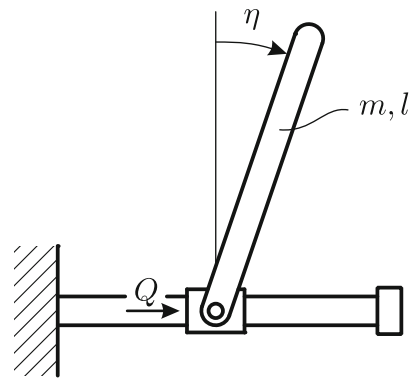

Fig. 5 Inverted pendulum

\subsection{Stabilizability of the inverted pendulum}

Stabilizability analysis of the inverted pendulum is presented according to [25]. Consider a second-order unstable system subjected to a digital PD controller in the form

$\ddot{\eta}(t)-a \eta(t)=-k_{\mathrm{p}} \eta\left(t_{j-1}\right)-k_{\mathrm{d}} \dot{\eta}\left(t_{j-1}\right), \quad t \in\left[t_{j}, t_{j+1}\right)$,

where $a>0$ is a system parameter and $k_{\mathrm{p}}$ and $k_{\mathrm{d}}$ are the proportional and the derivative control gains. If $a=6 \mathrm{~g} / \mathrm{l}$ then this system describes the well-known inverted pendulum subjected to the control force

$Q(t)=-\frac{m l}{6}\left(k_{\mathrm{p}} \eta\left(t_{j-1}\right)+k_{\mathrm{d}} \dot{\eta}\left(t_{j-1}\right)\right), \quad t \in\left[t_{j}, t_{j+1}\right)$,

where $l, m$ and $g$ denote the length and the mass of the pendulum and the gravitational acceleration, respectively (see Fig. 5).

We are looking for the critical system parameter $a_{\text {crit }}$, which limits stabilizability in the sense that if the system parameter is larger than the critical value then the system cannot be stabilized by the proposed PD controller at a fixed sampling period $\Delta t$. Introducing dimensionless time $T=\sqrt{a} t$ Eq. (12) can be rewritten as

$$
\begin{gathered}
\eta^{\prime \prime}(T)-\eta(T)=-K_{\mathrm{p}} \eta\left(T_{j-1}\right)-K_{\mathrm{d}} \eta^{\prime}\left(T_{j-1}\right), \\
T \in\left[T_{j}, T_{j+1}\right),
\end{gathered}
$$

where the dimensionless proportional and derivative control parameters are $K_{\mathrm{p}}=k_{\mathrm{p}} / a$ and $K_{\mathrm{d}}=k_{\mathrm{d}} / \sqrt{a}$, respectively. Using that the right-hand side of Eq. (14) is piecewise constant and by the matching the initial condition at time instant $T_{j}$, the solution can be written as

$$
\begin{aligned}
\eta(T)= & \left(\eta\left(T_{j}\right)+A\left(T_{j}\right)\right) \cosh \left(T-T_{j}\right) \\
& +\eta^{\prime}\left(T_{j}\right) \sinh \left(T-T_{j}\right)-A\left(T_{j}\right), \quad T \in\left[T_{j}, T_{j+1}\right),
\end{aligned}
$$

where $A\left(T_{j}\right)=-K_{\mathrm{p}} \eta\left(T_{j-1}\right)-K_{\mathrm{d}} \eta^{\prime}\left(T_{j-1}\right)$. Consequently, the controlled system can be described by the discrete map

$\mathbf{Y}\left(T_{j+1}\right)=\boldsymbol{\Phi} \mathbf{Y}\left(T_{j}\right)$, 
where $\mathbf{Y}\left(T_{j}\right)=\left(\begin{array}{lll}\eta\left(T_{j}\right) & \eta^{\prime}\left(T_{j}\right) & A\left(T_{j}\right)\end{array}\right)^{\mathrm{T}}$ and

$\boldsymbol{\Phi}=\left(\begin{array}{lll}\cosh (\Delta T) & \sinh (\Delta T) & \cosh (\Delta T)-1 \\ \sinh (\Delta T) & \cosh (\Delta T) & \sinh (\Delta T) \\ -K_{\mathrm{p}} & -K_{\mathrm{d}} & 0\end{array}\right)$,

which is actually the monodromy matrix of the system. The condition of the stability of the trivial solution is

$\left|\mu_{i}\right|<1, \quad i=1,2,3$,

where $\mu_{i}, i=1,2,3$ are the roots of the characteristic polynomial

$p(\mu)=\operatorname{det}(\mu \mathbf{I}-\boldsymbol{\Phi})$.

Using the Mobius transformation $\mu=(z+1) /(z-1)$, condition (18) can be transformed into

$\operatorname{Re}\left(z_{i}\right)<0, \quad i=1,2,3$,

where $z_{i}, i=1,2,3$ are the roots of the polynomial

$q(z)=(z-1)^{3} p\left(\frac{z+1}{z-1}\right)=b_{0}+b_{1} z+b_{2} z^{2}+b_{3} z^{3}$.

According to the Routh-Hurwitz criteria, this system is stable if $b_{1} / b_{3}>0$ and $b_{1} b_{2}-b_{0} b_{3}>0$. Using that $\Delta T>0$ the stable domain on the plane of control parameters is given by the conditions

$K_{\mathrm{p}}>1$

and

$$
\begin{aligned}
& -K_{\mathrm{p}}^{2} \tanh ^{2}\left(\frac{\Delta T}{2}\right)-K_{\mathrm{d}}^{2}+2 K_{\mathrm{p}} K_{\mathrm{d}} \tanh \left(\frac{\Delta T}{2}\right) \\
& -K_{\mathrm{p}} \tanh \left(\frac{\Delta T}{2}\right) \frac{2 \cosh (\Delta T)+1}{\sinh (\Delta T)} \\
& +K_{\mathrm{d}} \frac{2 \cosh (\Delta T)-1}{\sinh (\Delta T)}>0 .
\end{aligned}
$$

These conditions define a region in the plane $\left(K_{\mathrm{p}}, K_{\mathrm{d}}\right)$, which is bounded by a vertical line $K_{\mathrm{p}}=1$ and a parabola defined by Eq. (23). The condition for the existence of a stable domain is that the parabola intersects the line $K_{\mathrm{p}}=1$. As it can be seen in Fig. (6), the stable domain shrinks with increasing system parameter $a$ and it vanishes as $a$ exceeds a critical value

$a_{\text {crit }}=\left(\frac{1}{\Delta t} \operatorname{arcosh}\left(\frac{3}{2}\right)\right)^{2}$.

If $\Delta t=1$ then $a_{\text {crit }}=0.9263$. Thus, in case of $a=1$ there is no stable domain as it is shown in Fig. (6). The corresponding dimensionless parameters, where the stable domain disappears, are $K_{\mathrm{p} \text {, crit }}=1$ and $K_{\mathrm{d} \text {, crit }}=3 / \sqrt{5}$.

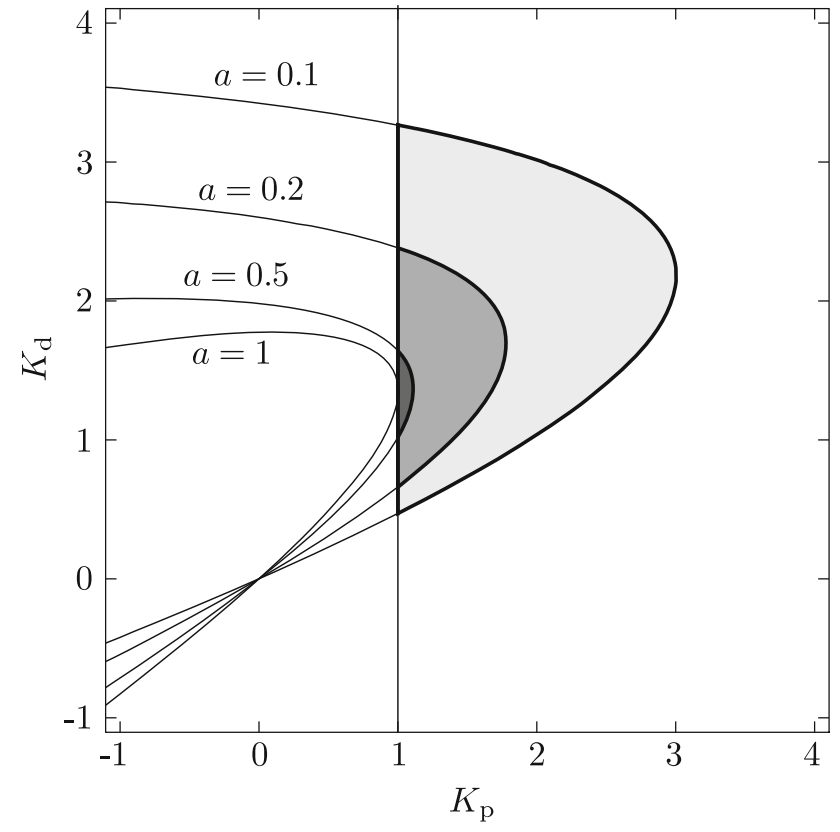

Fig. 6 Stability diagram for Eq. (14) for $\Delta t=1$ and for different system parameters $a$. Stable domains are indicated by grey shading

Consequently, the actual critical control gains are $k_{\mathrm{p} \text {, crit }}=$ $a_{\text {crit }}$ and $k_{\text {d,crit }}=3 \sqrt{a_{\text {crit }} / 5}$.

As it can be seen, stabilization is not possible if $a>a_{\text {crit }}$. Clearly, the frequency of the control loop is an important factor in the stabilization process, which is shown by the relation of $a_{\text {crit }}$ and $\Delta t$. In the next section, a similar analysis is performed for the turning process subjected to a digital PD controller described by Eq. (11).

\subsection{Stabilizability of the turning process}

Stability analysis of the hybrid system, given by Eq. (11), can be performed by the semi-discretization method [30]. The semi-discretization scheme is based on the discrete time scale $T_{i}=i h$, where $h$ is the discretization step determined as $\tau_{\mathrm{d}}=r h$ and $\Delta T=p h$ with $r$ and $p$ being integer approximation parameters. Here, we recall that Eq. (11) is actually a periodic system with principal period being equal to the sampling period $\Delta T$. Consequently, the integer $p$ is called the period resolution, since it is related to the resolution of the principal period. The integer $r$ is related to the discretization of the state over the delay interval $\left[T-\tau_{\mathrm{d}}, T\right]$, therefore it is called the delay resolution.

In the current analysis, the third-order two-point semidiscretization method is used according to [24,26]. In each discretization interval, the continuous-time delayed term $\eta\left(T-\tau_{\mathrm{d}}\right)$ is approximated by the linear combination of terms with piecewise-constant argument, namely, by $\eta\left(T_{i-r}\right), \eta^{\prime}\left(T_{i-r}\right), \eta\left(T_{i-r+1}\right)$ and $\eta^{\prime}\left(T_{i-r+1}\right)$. This way, the delayed system is approximated by a series of ODEs, and a 
monodromy map can be constructed over the sampling period $\Delta T$. The size of the approximate monodromy matrix is determined by the delay resolution $r$, while the period resolution $p$ determines the number of matrix multiplications within one sampling period. Consequently, computational costs increase with increasing $r$ and $p$. The steps of the semi-discretization method for hybrid systems such as Eq. (11) are given in $[24,26]$.

Figure 7 shows a series of stability diagrams in the plane $\left(K_{\mathrm{p}}, K_{\mathrm{d}}\right)$ for different dimensionless spindle speeds $\Omega_{\mathrm{d}}$ and dimensionless depth of cut $H_{\mathrm{d}}$. These diagrams are actually projections of the 4 dimensional stability chart in the parameter space $\left(K_{\mathrm{p}}, K_{\mathrm{d}}, \Omega_{\mathrm{d}}, H_{\mathrm{d}}\right)$. The case $H_{\mathrm{d}}=0$ corresponds to the equation

$$
\begin{aligned}
\eta^{\prime \prime}(T)+2 \zeta \eta^{\prime}(T)+\eta(T)= & -K_{\mathrm{p}} \eta\left(T_{j-1}\right)-K_{\mathrm{d}} \eta^{\prime}\left(T_{j-1}\right), \\
& T \in\left[T_{j}, T_{j+1}\right),
\end{aligned}
$$

which is similar to Eq. (14), only the coefficients of the terms $\eta(T)$ and $\eta^{\prime}(T)$ are different. Consequently, the corresponding stability diagrams are similar to the one shown in Fig. 6, but here the vertical stability boundary is at $K_{\mathrm{P}}=-1$. Actually, the stability boundary at $K_{\mathrm{P}}=-1$ plays an important role for the cases $H_{\mathrm{d}}>0$, since the stability domain disappears next to this line. Comparison to the SLD of the uncontrolled turning process in Fig. 4 shows that the depth of cut can significantly be increased without chatter by properly tuned control gains. For instance, if $\Omega_{\mathrm{d}}=1.5$ then the system can be stabilized for $H_{\mathrm{d}}=1.5$, but for $H_{\mathrm{d}}=2$ it is unstable for any $K_{\mathrm{p}}$ and $K_{\mathrm{d}}$. This implies that the critical dimensionless depth of cut $H_{\mathrm{d} \text {, crit }}$ limiting the stabilizability is between 1.5 and 2 . In the next section, the exact value of $H_{\mathrm{d} \text {,crit }}$ is determined, and the stabilizability diagram is constructed.

\section{Stabilizability diagram}

Stabilizability diagram presents the critical dimensionless depth of cut $H_{\mathrm{d} \text {,crit }}$ versus the dimensionless spindle speed $\Omega_{\mathrm{d}}$. If $H_{\mathrm{d}}<H_{\mathrm{d} \text {,crit }}$ then the machining process can be stabilized by a properly tuned digital PD controller, but if $H_{\mathrm{d}}>H_{\mathrm{d} \text {, crit }}$ the system cannot be stabilized. This diagram can directly be compared to the traditional SLD shown in Fig. 4.

The stabilizability diagram is determined according to the following steps.

1. The dimensionless sampling period $\Delta T$ is fixed. Note that in the previous papers $[24,26]$, the stability diagrams were determined for the special cases where the regenerative delay is integer multiple of sampling period. Here, the sampling period is independent of the regenerative delay.
2. The investigated region of the dimensionless spindle speed $\left[\Omega_{\mathrm{d}, \min }, \Omega_{\mathrm{d}, \max }\right]$ is divided up into $(n-1)$ nonequidistant intervals by discrete values $\Omega_{\mathrm{d}, i}, i=1,2$, $\ldots, n$ such that the ratio of the corresponding dimensionless regenerative delay $\tau_{\mathrm{d}, i}=2 \pi / \Omega_{\mathrm{d}, i}$ and the dimensionless sampling period $\Delta T$ is a rational number, i.e, $\tau_{\mathrm{d}, i} / \Delta T=r_{i} / p_{i}$, where $r_{i}, p_{i} \in \mathbb{Z}^{+}$give the delay and the period resolutions, respectively. The resolution of the axis $\Omega_{\mathrm{d}}$ was such that $\Omega_{\mathrm{d}, i+1}-\Omega_{\mathrm{d}, i}<$ $\frac{2}{n}\left(\Omega_{\mathrm{d}, \text { max }}-\Omega_{\mathrm{d}, \text { min }}\right)$ and $r_{i} \geq 30$ for all $i=1,2, \ldots, n$. According to previous studies [24], this resolution provides a good approximation in the investigated parameter region (along the first 5 lobes).

3. The spindle speed is fixed step-by-step to the discrete values $\Omega_{\mathrm{d}}=\Omega_{\mathrm{d}, i}, i=1,2, \ldots, n$. For each fixed $\Omega_{\mathrm{d}}=$ $\Omega_{\mathrm{d}, i}$, the semi-discretization step is determined as $h_{i}=$ $\tau_{\mathrm{d}, i} / r_{i}$.

4. The investigated region of the dimensionless depth of cut $\left[H_{\mathrm{d}, \min }, H_{\mathrm{d}, \max }\right]$ is divided up into $(m-1)$ equidistant initial intervals by discrete values $H_{\mathrm{d}, j}=j \Delta H_{\mathrm{d}}, j=$ $1,2, \ldots, m$ with $\Delta H_{\mathrm{d}}=\left(H_{\mathrm{d}, \max }-H_{\mathrm{d}, \min }\right) / m$. The stability diagrams in the plane $\left(K_{\mathrm{p}}, K_{\mathrm{d}}\right)$ are determined for all $H_{\mathrm{d}}=H_{\mathrm{d}, j}, j=1,2, \ldots, m$. If the case $H_{\mathrm{d}}=H_{\mathrm{d}, j}$ can be stabilized but there is no stable domain for $H_{\mathrm{d}}=$ $H_{\mathrm{d}, j+1}$, then the interval $\left[H_{\mathrm{d}, j}, H_{\mathrm{d}, j+1}\right]$ is further investigated by interval halving method, i.e., stability diagrams are determined for $H_{\mathrm{d}}=H_{\mathrm{d}, j}+\frac{1}{2} \Delta H_{\mathrm{d}}$, then either for $H_{\mathrm{d}}=H_{\mathrm{d}, j}+\frac{1}{4} \Delta H_{\mathrm{d}}$ or for $H_{\mathrm{d}}=H_{\mathrm{d}, j}+\frac{3}{4} \Delta H_{\mathrm{d}}$, etc. The procedure is stopped if $H_{\mathrm{d}}$ is given by an accuracy of $\Delta H_{\mathrm{d}} / 2^{7}$ and its value is recorded as $H_{\mathrm{d}, \text { crit }}$.

5. The critical value $H_{\mathrm{d}, \text { crit }}$ is determined for all discrete spindle speeds $\Omega_{\mathrm{d}, i}, i=1,2, \ldots, n$ and the stabilizability diagram is plotted.

Figure 8 presents the stabilizability diagram for the dimensionless sampling period $\Delta T=0.5$ and damping ratio $\zeta=$ 0.05 . The boundaries of the investigated parameter domain are $\left[\Omega_{\mathrm{d}, \min }, \Omega_{\mathrm{d}, \max }\right]=[0.2,2.5]$ and $\left[H_{\mathrm{d}, \min }, H_{\mathrm{d}, \max }\right]=$ [0,3.5] with $n=100, m=4$. The actual sampling period is $\Delta t=\Delta T / \omega_{\mathrm{n}}=0.5 / \omega_{\mathrm{n}}$, i.e,. the digital control samples the state at a frequency $f=1 / \Delta t=2 \omega_{\mathrm{n}}$, which is the double of the natural frequency of the system. The traditional stability lobe diagram (i.e., the uncontrolled case) is also shown by dashed line. It can be seen the depth of cut can significantly be increased. At the resonant spindle speed $\Omega_{\mathrm{d}}=1 / k, k=1,2, \ldots$ the critical dimensionless depth of cut is about the double of the traditional SLD, while in between the resonant spindle speeds, the depth of cut is increased by a factor of $8-10$. Note that this gain is achieved in spite of the fact that sampling frequency of the controller is relatively low compared to natural frequency of the uncontrolled system $\left(f=2 \omega_{n}\right)$. 
Fig. 7 Stability diagrams for different $\Omega_{\mathrm{d}}$ and $H_{\mathrm{d}}$ values. Stable domains are indicated by gray shading. Empty plots indicate that the system cannot be stabilized

Fig. 8 Stabilizability diagram for a turning process with $\zeta=0.05$ subjected to a digital PD control with sampling frequency $f=2 \omega_{\mathrm{n}}(\Delta T=0.5)$ (a), the associated critical proportional gain (b) and the associated critical derivative gain (c)
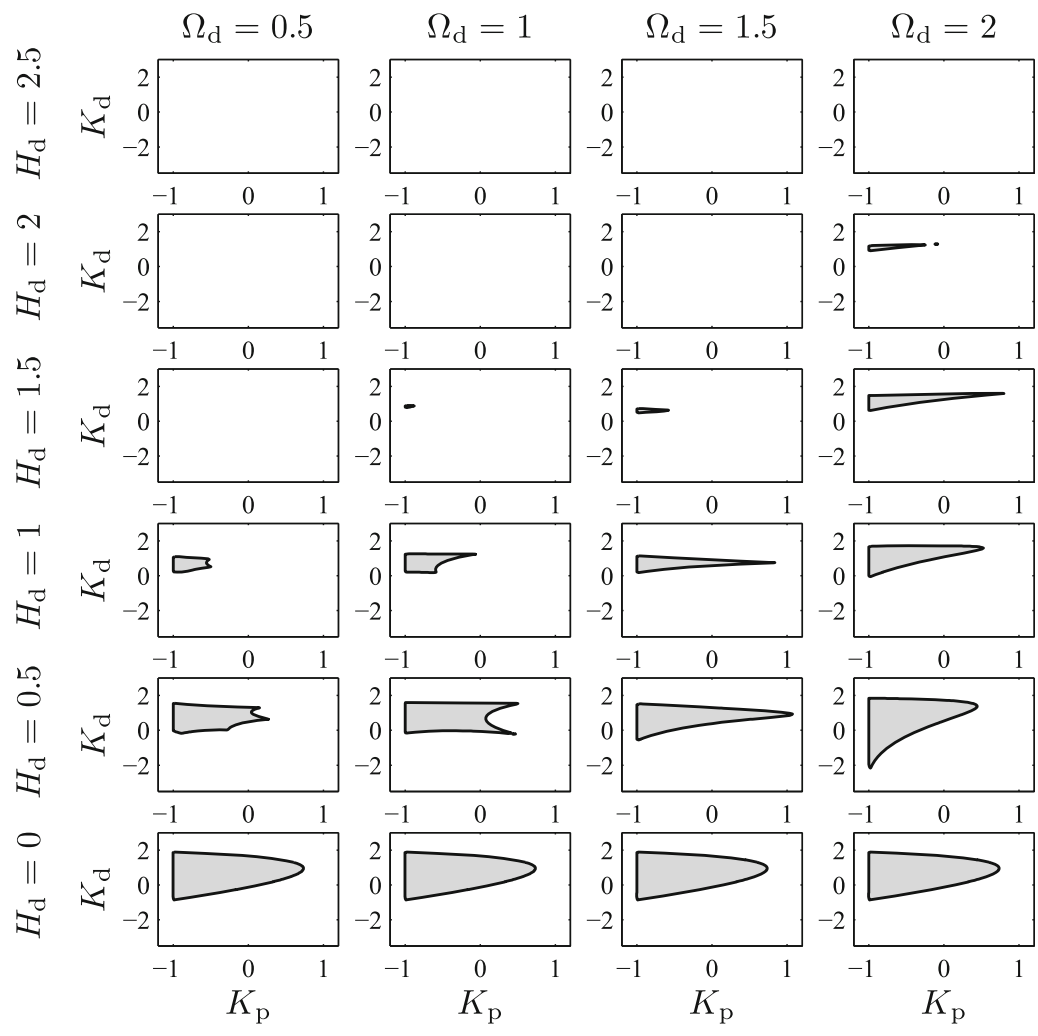

$\Omega_{\mathrm{d}}=2.5$
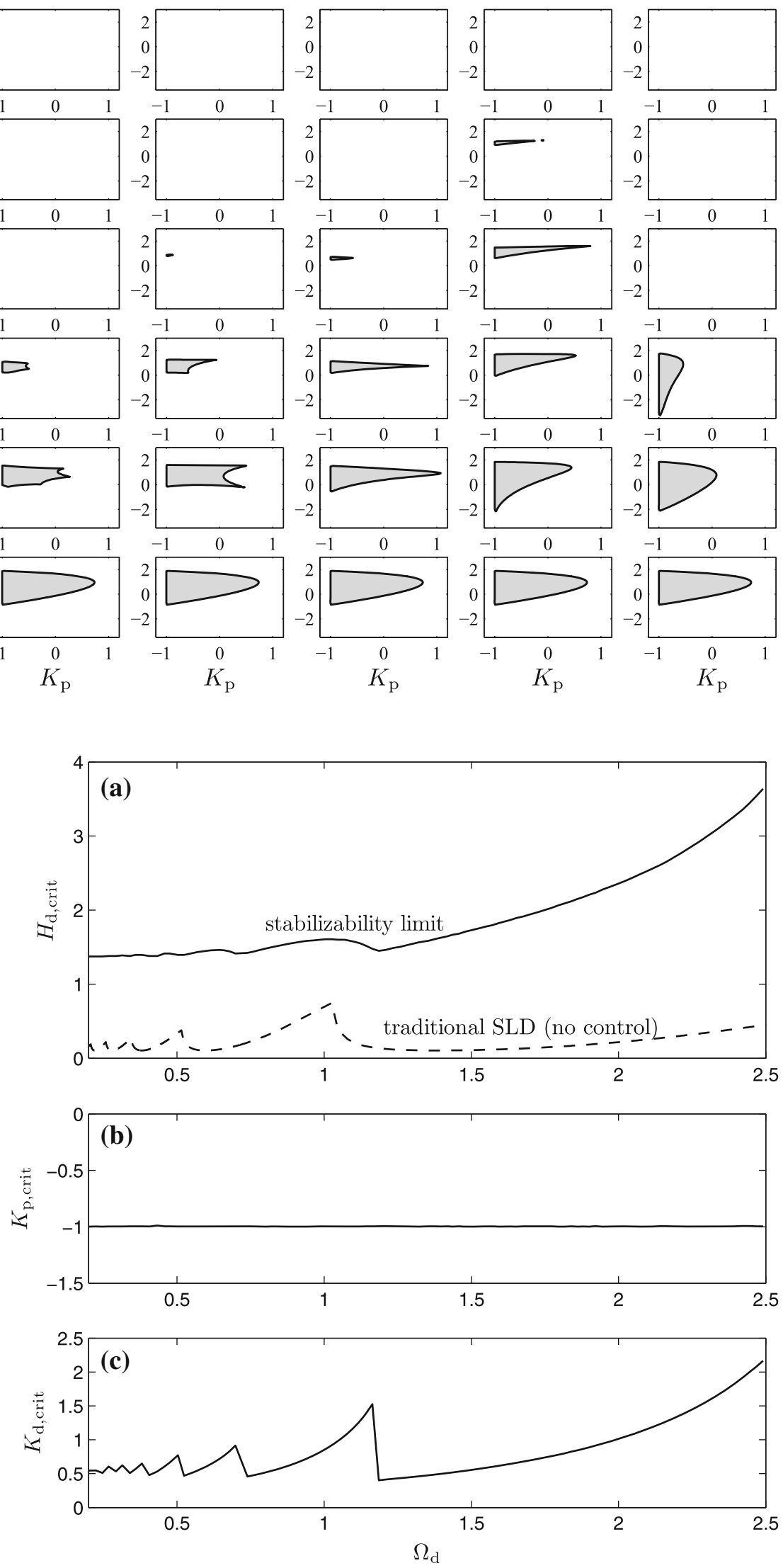
Figure 8 also presents the critical dimensionless proportional and derivative control gains that are associated with the disappearance of the stability domain in the plane $K_{\mathrm{p}}, K_{\mathrm{d}}$. Note that $K_{\text {p,crit }}=-1$, which means that the stability domain disappears at the stability limit $K_{\mathrm{p}}=-1$ as it can also be observed in Fig. 7.

\section{Conclusion}

Stabilizability of turning processes subjected to digital PD controller was analyzed with special attention to the discrete nature of the controller such as sampling effect and digital delay. It was shown that for a reasonable sampling period the depth of cut can be increased by a factor 2-10 even in the case when the sampling frequency of the controller is just the double of the natural frequency of the system. The stabilizing control gains were also given.

Note that here we presented an ideal stabilizability analysis in the sense that we assumed that the state output is available for the feedback without any noise and uncertainties, and the parameters of the machining system are also perfectly known at all time instances. In practice, measured output is affected by noise and the system parameters are changing during the operation due changing machining conditions (e.g., tool wear, changes in the local temperature on the active face of the tool, etc.). Intuitively, if the uncertainties in the system parameters and the output were also taken into account then the gain in the maximal depth of cut would decrease. Actually, consideration of these uncertainties requires a robust stabilizability analysis with respect to perturbation in the system parameters and the output.

In spite of these modelling restrictions, the current analysis still presents a general view on the stabilizability of machining operation. It was shown that the interplay between the regenerative delay and the digital effects of the controller may strongly affect the stabilizability of the system.

Acknowledgments The authors gratefully acknowledge support from the Hungarian National Science Foundation under Grant OTKAK105433. This research was supported by the European Union and the State of Hungary, co-financed by the European Social Fund in the framework of TÁMOP 4.2.4. A/-11-1-2012-0001 "National Excellence Program".

\section{References}

1. Tlusty J, Polacek A, Danek C, Spacek J (1962) Selbsterregte Schwingungen an Werkzeugmaschinen. VEB Verlag Technik, Berlin

2. Tobias SA (1965) Machine tool vibration. Blackie, London 1965

3. Altintas Y (2000) Manufacturing automation: metal cutting mechanics, machine tool vibrations, and $\mathrm{CNC}$ design. Cambridge University Press, New York
4. Quintana G, Ciurana J (2011) Chatter in machining processes: A review. Int J Mach Tools Manuf 51:363-376

5. van Dijk NJM, Doppenberg EJJ, Faassen RPH, van de Wouw N, Oosterling JAJ, Nijmeijer H (2010) Automatic in-process chatter avoidance in the high-speed milling process. J Dyn Syst Meas Control 132:031006-031011

6. Dombovari Z, Altintas Y, Stepan G (2010) The effect of serration on mechanics and stability of milling cutters. Int J Mach Tools Manuf 50:511-520

7. Bachrathy D, Stepan G (2013) Improved prediction of stability lobes with extended multi frequency solution. CIRP Ann Manuf Technol 62:411-414

8. Jayaram S, Kapoor SG, DeVor RE (2000) Analytical stability analysis of variable spindle speed machining. J Manuf Sci Eng 122:391-397

9. Zatarain M, Bediaga I, Munoa J, Lizarralde R (2008) Stability of milling processes with continuous spindle speed variation: analysis in the frequency and time domains, and experimental correlation. CIRP Ann Manuf Technol 57:379-384

10. Seguy S, Insperger T, Arnaud L, Dessein G, Peigne G (2010) On the stability of high-speed milling with spindle speed variation. Int J Adv Manuf Technol 48:883-895

11. Otto A, Radons G (2013) Application of spindle speed variation for chatter suppression in turning. CIRP J Manuf Sci Technol 6(2):102-109

12. Tarng YS, Kao JY, Lee EC (2000) Chatter suppression in turning operations with a tuned vibration absorber. J Mater Process Technol 105:55-60

13. Sims ND (2007) Vibration absorbers for chatter suppression: a new analytical tuning methodology. J Sound Vib 301:592-607

14. Yang Y, Munoa J, Altintas Y (2010) Optimization of multiple tuned mass dampers to suppress machine tool chatter. Int J Mach Tools Manuf 50:834-842

15. Gourc E, Seguy S, Michon G, Berlioz A (2013) Chatter control in turning process with a nonlinear energy sink. Adv Mater Res 698:89-98

16. Shiraishi M, Yamanaka K, Fujita H (1991) Optimal control of chatter in turning. Int J Mach Tools Manuf 31(1):31-43

17. Ganguli A, Deraemaeker A, Preumont A (2007) Regenerative chatter reduction by active damping control. J Sound Vib 300: 847-862

18. Munoa J, Mancisidor I, Loix N, Uriarte LG, Barcena R, Zatarain M (2013) Chatter suppression in ram type travelling column milling machines using a biaxial inertial actuator. CIRP Ann Manuf Tech 62(1):407-410

19. Chen M, Knospe CR (2007) Control approaches to the suppression of machining chatter using active magnetic bearings. IEEE Trans Control Syst Technol 15:220-232

20. van Dijk NJM, van de Wouw N, Doppenberg EJJ, Oosterling HAJ, Nijmeijer H (2012) Robust active chatter control in the high-speed milling process. IEEE Trans Control Syst Technol 20(4):901-917

21. Zhang Y, Sims ND (2005) Milling workpiece chatter avoidance using piezoelectric active damping: a feasibility study. Smart Mater Sturct 14:N65-N70

22. Long X, Jiang H, Meng G (2013) Active vibration control for peripheral milling processes. J Mater Process Technol 21:660-670

23. Brecher C, Schulz A (2005) Electrohydraulic active damping system. CIRP Ann 54(1):389-392

24. Lehotzky D, Insperger T (2012) Stability of turning processes subjected to digital PD control. Per Pol Mech Eng 56(1):33-42

25. Enikov E, Stepan G (1998) Micro-chaotic motion of digitally controlled machines. J Vib Control 2:427-443

26. Lehotzky D, Insperger T (2014) Stability of systems with state delay subjected to digital control. In: Vyhlidal T, Lafay J-F, Sipahi $\mathrm{R}$ (eds) Delay systems - from theory to numerics and applications. Springer, Switzerland, pp 71-84 
27. Insperger T, Stepan G, Turi J (2007) State-dependent delay in regenerative turning processes. Nonlinear Dyn 47:275-283

28. Wiercigroch M, Budak E (2000) Sources of nonlinearities, chatter generation and suppression in metal cutting. Philos Trans Roy Soc A 359:663-693

29. Insperger T, Stepan G (2002) Semi-discretization method for delayed systems. Int J Numer Methods Eng 55:503-518

30. Insperger T, Stepan G (2011) Semi-discretization for time-delay systems. Springer, New York

31. Budak E, Altintas Y (1998) Analytical prediction of chatter stability in milling, part I: general formulation. J Dyn Syst 120: 22-30

32. Breda D, Maset S, Vermiglio R (2012) Approximation of eigenvalues of evolution operators for linear retarded functional differential equations. SIAM J Numer Anal 50(3):1456-1483
33. Sun JQ, Song B (2009) Control studies of time-delayed dynamical systems with the method of continuous time approximation. Commun Nonlinear Sci 14:3933-3944

34. Zhang X-Y, Sun J-Q (2013) A note on the stability of linear dynamical systems with time delay. J Vib Control. doi:10.1177/ 1077546312473319

35. Khasawneh FA, Mann BP (2011) A spectral element approach for the stability of delay systems. Int J Numer Methods Eng 87(6):566592

36. Sieber J, Szalai R (2011) Characteristic matrices for linear periodic delay differential equations. SIAM J Appl Dyn Syst 10(1):129-147

37. Lampe BP, Rosenwasser EN (2011) Stability investigation for linear periodic time-delayed systems using Fredholm theory. Autom Remote Control 72(1):38-60

38. Stepan G (1989) Retarded dynamical systems. Longman, Harlow 